\section{RESEARCH PERSPECTIVES}

\author{
A.R. Borges \\ P. Lopez-Larrubia \\ J.B. Marques \\ S.G. Cerdan
}

\section{MR Imaging Features of High-Grade Gliomas in Murine Models: How They Compare with Human Disease, Reflect Tumor Biology, and Play a Role in Preclinical Trials}

\begin{abstract}
SUMMARY: Murine models are the most commonly used and best investigated among the animal models of HGG. They constitute an important weapon in the development and testing of new anticancer drugs and have long been used in preclinical trials. Neuroimaging methods, particularly MR imaging, offer important advantages for the evaluation of treatment response: shorter and more reliable treatment end points and insight on tumor biology and physiology through the use of functional imaging DWI, PWI, BOLD, and MR spectroscopy. This functional information has been progressively consolidated as a surrogate marker of tumor biology and genetics and may play a pivotal role in the assessment of specifically targeted drugs, both in clinical and preclinical trials. The purpose of this Research Perspectives was to compile, summarize, and critically assess the available information on the neuroimaging features of different murine models of HGGs, and explain how these correlate with human disease and reflect tumor biology.
\end{abstract}

A nimal models of HGG have become an imperative tool in the development and testing of new anticancer drugs. The combination of these advanced neuroimaging methods provides a novel integrated environment in which a collection of noninvasive biomarkers validated on well-defined animal models may provide important clues to tumor biology and response to treatment, with potential applications in human neoplasms. In general, the use of animal models overcomes many of the limitations found in the clinical setting, including impracticable serial tissue sampling and difficulties in establishing correlations between tumor physiopathology and its genetic profile, a circumstance limiting considerably the successful implementation of tailored personalized cancer treatments.

Received September 13, 2011; accepted after revision October 11.

From the Radiology Department (A.R.B.), Instituto Português de Oncologia de Lisboa, Lisboa, Portugal; Laboratory for Imaging and Spectroscopy by Magnetic Resonance (A.R.B., P.L.-L., S.G.C.), Instituto de Investigaciones Biomédicas "Alberto Sols," Madrid, Spain; Programme for Advanced Medical Education (A.R.B.), Champalimaud and Calouste Gulbenkian Foundations, Lisbon, Portugal; and Neurology Department (J.B.M.), Instituto Português de Oncologia de Lisboa, Lisbon, Portugal.

This work was supported by the Programme for Advanced Medical Education from Fundação Champalimaud, Fundação Calouste Gulbenkian, Ministério da Saúde and Fundação para a Ciência e Tecnologia, Portugal, to the first author (A.R.B.), and by grants from the Spanish Ministry of Science and Innovation SAF 2008-01327 and the Community of Madrid S-BI0-2006-0170, to the last author (S.G.C.)

Please address correspondence to A.R. Borges, MD, Instituto Portugues de Oncologia de Lisboa, Radiology, Rua Professor Lima Basto, Lisbon, 1093, Portugal; e-mail: borgalexandra@gmail.com, aborges@iib.uam.es

Indicates open access to non-subscribers at www.ajnr.org

Indicates article with supplemental on-line tables.

http://dx.doi.org/10.3174/ajnr.A2959
Among neuroimaging methods, MR imaging is the most common technique to assist the management of patients with brain tumors; it is free of ionizing radiation, overall well-tolerated, and provides detailed anatomic and functional information. Neuroimaging features of murine models of HGG have been less explored due to technical limitations. Highresolution MR imaging of small animals requires high-field magnets with small bores and dedicated coils, adequate anesthesia, and tailored fixation devices (Fig 1).

Murines are the most commonly used and are the best investigated models of human glioma. They are easy to handle, have a short life span, and develop central nervous system tumors that are similar to their human counterparts. ${ }^{1,2}$ Moreover, the genetic information currently available on the Mouse Genome Project and the technologic developments in genetic manipulation (transgenics, knockouts, and conditional knockouts) make this model very appealing for unraveling HGG biology. ${ }^{2}$

This article provides an overview of the currently available murine models of HGG and of the wide gamut of neuroimaging methodologies that can be used to characterize their morphology, physiology, and biology. We will specifically focus on MR imaging, emphasizing conventional and functional techniques and their potential applications in clinical and preclinical trials.

\section{Murine Models of HGG}

A wide range of murine models of HGG are available, each one having different advantages and drawbacks. ${ }^{1}$ These models can be broadly divided into endogenous and exogenous, depending whether the tumor originates in the primary host or is grafted or implanted into the host. Endogenous models in- 

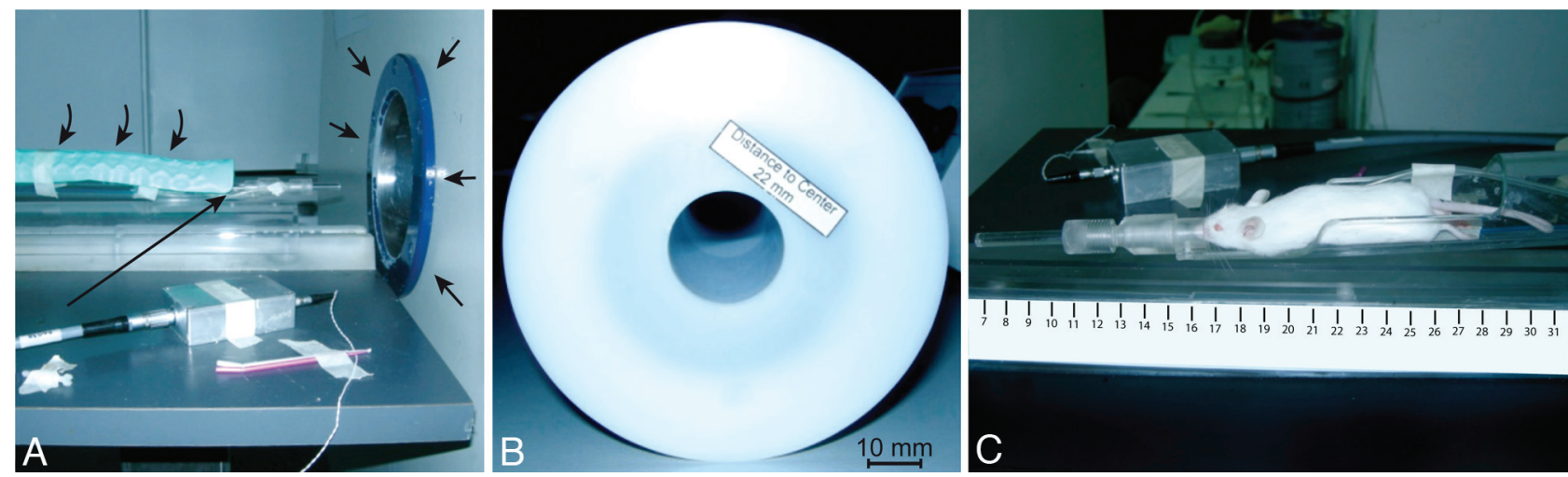

Fig 1. Dedicated MR imaging magnet for small animals and scanning devices. A, 7.4T Bruker Pharmascan (Bruker BioSpin, Rheinstetten, Germany) with a 160-mm horizontal bore (small arrows). A dedicated fixation device for mice brain studies is seen in place, equipped with continuous isofluorane anesthesia (/ong arrow), respiratory monitoring, and a thermostatic water mat set for $37^{\circ} \mathrm{C}$ (curved arrows). B, Dedicated mouse head coil (22-mm diameter). C, In-house built poly-methyl-methacrylate bed and fixation device with a Swiss mouse in place.

clude spontaneous carcinogenesis in inbred strains, carcinogenic-induced tumors by intrauterine or postnatal exposure to radiation, chemical or infectious mutagenic agents, and genetically engineered tumor models obtained by introducing genetic changes known to promote carcinogenesis in humans. Exogenous models require grafting or implantation of a suspension of tumor cells, tumor explants, or tumor spheroids from primary or stable tumor cell lines into the host animal. Whereas endogenous models more accurately reproduce and recapitulate the biologic behavior and the complex relationship between the tumor and its microenvironment, grafts have a lower cost and a more predictable and reproducible behavior in terms of tumor take and time for tumor development, making them more appealing for preclinical trials. ${ }^{2,3}$

Graft models may be syngeneic when the tumor and host have the same origin (allograft) or heterogeneic when they have different origins (xenograft). Human xenografts recapitulate the human neoplasm but are not representative of the parent tumor-host interaction because the tumor and microenvironment have different origins. ${ }^{4}$ Moreover, they require the use of nude mice, departing further from the actual clinical situation.

According to the grafted or implanted site, models are subdivided into orthotopic (tumor implanted in its parent organ) or heterotopic (tumor implanted in a different organ). Orthotopic models are representative of the tumor environment, but in the brain, they are of difficult clinical access requiring imaging to assess tumor development and growth. In most heterotopic models, tumors are implanted in the subcutaneous soft tissues where clinical assessment is easy and immediate, but unfortunately they do not represent the parent tumormicroenvironment interaction.

Tumor explants from actual patients may be implanted in mice in the form of grafts, spheroids, or primary cell lines, providing specific information on tumor behavior and tumor response to different treatment regimens, serving as an invaluable tool for personalized oncologic management. ${ }^{5}$ Grafting tumor fragments by microsurgical techniques avoids the artificial diffusion of intracerebral injections of cell suspensions. ${ }^{4,5}$ Engraftment of primary glioblastoma biopsies has shown high take rates but a long average latency. ${ }^{6}$ The use of primary tissue prevents the genetic drift and phenotypic changes secondary to prolonged passages in vitro, reproduces the phenotypic traits of the native tumor, and displays more cellular heterogeneity than established cell lines. ${ }^{6}$ The use of spheroids (3D cultures), as opposed to cell suspensions (2D cultures), avoids the disruption of the stromal compartment preserving the original tumor environment. ${ }^{6}$

Many HGG cell lines of human and murine origin are currently available and accurately reproduce the histologic and cytologic features of human neoplasms. ${ }^{1,7,8}$ Cell lines of recurrent tumors that reproduce the aggressive behavior and treatment resistance of clinical recurrences are also available. ${ }^{9}$ These can be obtained directly from a recurrent tumor; from an enriched lung metastasis assay, or from sorting tumor cells, with specific markers of aggressiveness, by using fluorescenceactivated cell sorting. ${ }^{9}$ Furthermore, it is possible to isolate and purify brain tumor-initiating cells that give rise to highly resistant neoplasms. ${ }^{10}$

GEMMs recapitulate the genetic events of human neoplasms, provide an increased understanding of brain tumor biology, and help the development of treatment strategies that target specific oncogenic pathways. ${ }^{8,11,12}$ In these models, the initiating genetic event is known; mice spontaneously develop orthotopic tumors and are immunocompetent. ${ }^{12}$ They can be used for screening and target validation and to evaluate the efficacy and potential off-target effects of new drugs. ${ }^{12}$ For limiting tumor development to local somatic cells, gene delivery systems based on viral vectors and transgenic receptors under specific promoters (glial fibrillary acid protein to hit astrocytes and nestin to hit glioneuronal progenitors) are now widely available. The low transfection efficiency and the need for secondary stochastic genetic events for tumor onset lead to low tumor take and unpredictable tumor latency. These were the major problems limiting the use of GEMMs in wide-scale high-throughput preclinical trials. ${ }^{8,10,11,13}$ However, breeding transgenic mice with knockout mice with targeted deletions or mutations in tumor-suppressor genes leads to malignant transformation at a faster and more predictable rate, reflecting both the induction and progression of tumorigenesis. ${ }^{8,12,13}$

More recently, transplantable models derived from GEMMs combine the advantages of both endogenous and exogenous models: high penetrance; rapid and predictable tumor growth; use of syngeneic immunocompetent host; and reproduction of the genetic, molecular, and histologic features of human parental tumors. ${ }^{11}$ 


\begin{tabular}{l} 
Table 1: Distinctive features of the $\mathbf{2}$ main groups of HGG mouse \\
models \\
\hline Features \\
\hline Endogenous models (GEMMs and nontargeted mutagenesis/carcinogenesis) \\
Unpredictable latency periods, growth curves, tumor location, and \\
morphology; implies regular screening for tumor detection and longitudinal \\
monitoring for progression; more physiologic and best representing human \\
disease \\
Exogenous models or xenografts \\
Predictable and reproducible, high tumor take; best for high throughput \\
preclinical trials; less physiologic and less representative of the actual \\
human condition
\end{tabular}

In summary, a valid brain tumor model should fulfill the following criteria: 1) derive from glial cells, 2) grow as a stable cell line, 3) propagate in vivo by serial transplantation, 4) have a predictable and reproducible tumor take and growth rate, 5) maintain gliomalike features, 6) have a predictable host survival (long enough to allow the assessment of treatment effect), 7) be not or weakly immunogenic in the syngeneic host, 7) have no extent beyond the brain, and 10) have a response to conventional treatment, or lack of it, predicting the behavior of the human counterpart. ${ }^{10}$

The main features of the currently available mouse models of HGG are summarized in Tables 1 and 2.

\section{Neuroimaging Modalities Applied to Murine Models of HGG: Why and How}

Neuroimaging methods, as applied to mouse models of HGG, constitute a powerful tool to guide the development, test the efficacy, and monitor the effect of new drugs in preclinical trials. They allow the use of smaller animal cohorts, shorten the duration of studies (by setting more efficient end points for treatment response than survival), and provide serial longitudinal information in vivo about tumor physiology and biology. ${ }^{14}$ In short, neuroimaging is placed ideally to become a longitudinal biomarker in clinical and preclinical testing.

Many different imaging modalities have been proposed for imaging mice: optical imaging (bioluminescence imaging, fluorescence, and near-infrared optical imaging), CT, MR imaging, MR spectroscopy, SPECT, and PET, providing different and often complementary information. ${ }^{15}$ More recently, multimodality imaging combines the advantages of different imaging modalities to get a fully integrated picture of tumor behavior and of neurotherapeutic effects: PET/CT, PET-MR, fluorescent molecular tomography, MR bioluminescence imaging. ${ }^{15}$ With the exception of optical imaging, all other imaging modalities can be easily translated to human disease. Fusion of these imaging modalities allows in vivo visualization of trifusion reporter genes labeled with different markers depicted by different imaging techniques.

On clinical grounds, MR imaging is used to diagnose, assess disease extent, guide treatment decisions, evaluate response over time, and define patient prognosis. ${ }^{16}$ Existing protocols allow a range of both structural and functional information to be drawn from imaging datasets: Imaging phenotypes can be defined, quantified, and compared with the biologic and genetic profiling of tumors (Fig 2). ${ }^{17,18}$

The most important diagnostic and prognostic features of a tumor involve its perfusion status, oxygenation, and glucose consumption. At the present time, MR imaging can render information on tumor burden, cell density, vascularization, tumor oxygenation, and metabolism through the use of functional sequences such as DWI, PWI, BOLD and tissue oxygen level-dependent imaging, and MR spectroscopy. These provide high-resolution parametric mapping with an easy readout for treatment efficacy and longitudinal drug monitoring (Fig 3). ${ }^{16}$ Multispectral analysis techniques can provide automated differences between viable and necrotic, highly proliferative versus quiescent, highly vascularized versus poorly vascularized, and hypoxic versus normoxic tumor tissue. Therefore, MR imaging phenotypes provide indirect mapping of gene expression and can be used to select specific tumor regions for genomic analysis or for targeted oncotherapy. Different tumor phenotypes have already been identified in humans and correlated well with different patterns of gene expression: angiogenic and poorly invasive, angiogenic and invasive, and nonangiogenic invasive phenotypes. ${ }^{16,19}$

Adapting human protocols to the mouse scale involves many challenges: obtaining images with similar resolution and signal intensity-to-noise ratios when facing a 10- to 15 -fold decrease in voxel size on each dimension, increasing image throughput for large-scale screening, and analyzing anatomic images and parametric data from multiple mouse datasets. ${ }^{16}$

MR imaging of mice can be performed in clinical scanners with or without dedicated radio-frequency coils or in dedicated small-bore high-field-strength scanners (now as high as 17.6T). ${ }^{16}$

Mouse imaging can be performed in vivo or postmortem and in series (a single animal at a time) or in parallel (by scanning various animals simultaneously, also known as multiple mouse MRI, Fig 4). ${ }^{20}$

Postmortem. MR imaging provides high-resolution images by reducing unavoidable pulsatile movements, allowing strict fixation, very long scanning times, and the use of high concentrations of gadolinium. ${ }^{16}$ The excellent correlation with pathology sections justifies the eponym "MR histology" and provides in situ phenotypic information. Postmortem imaging can be easily added to in vivo studies at the end of the follow-up time to guide conventional histologic analysis. With an 11.7T MR imaging scanner and scanning times up to 37 hours, an in-plane resolution of $20 \mu \mathrm{m}$ can be achieved. ${ }^{16} \mathrm{In}$ the Visible Mouse Project, FSE T2-weighted images with a $100-\mu \mathrm{m}$ isotropic resolution were produced within a reasonable timeframe ( 2 hours 45 minutes) (Fig 5). ${ }^{16,21}$

The power of preclinical trials increases with the increasing number of animals, requiring an intervention and a control group. Therefore, a high image throughput and an efficient and standardized imaging protocol are essential if one aims to use MR imaging end points. Imaging of single mice is expensive and time-consuming and exhausts scanner availability for research. Multiple mouse MRI overcomes most of these problems. ${ }^{22}$ Virtually, any clinical scanner can be used without modification, though at the cost of poor resolution and image degradation. Alternative magnet designs have been developed for this purpose, each with its own advantages and disadvantages. Large bore, large gradient, and large radio-frequency coil magnets can be used to image several mice within the same FOV, but labor intensive postprocessing is required to separate datasets of individual mice. The use of a common gradient 


\begin{tabular}{|c|c|c|c|c|c|c|}
\hline Animal Model/Subtype & Immunogenicity & $\begin{array}{c}\text { Reproducible } \\
\text { Tumor-Host } \\
\text { Interaction }\end{array}$ & $\begin{array}{c}\text { Tumor Take } \\
\text { and Growth } \\
\text { Rate }\end{array}$ & $\begin{array}{c}\text { Reproducibility } \\
\text { of Human } \\
\text { HGG } \\
\text { Genetics and } \\
\text { Biology }\end{array}$ & $\begin{array}{l}\text { Stromal } \\
\text { Disruption }\end{array}$ & $\begin{array}{c}\text { High- } \\
\text { Throughput } \\
\text { Preclinical } \\
\text { Trials }\end{array}$ \\
\hline \multicolumn{7}{|l|}{ Exogenous models or xenografts } \\
\hline Syngeneic & None/low & & & & & Yes \\
\hline Heterogeneic & High/requires nude mice & & & & & Yes \\
\hline Orthotopic & & Yes & & & & Yes \\
\hline Heterotopic & & No & & & & Yes \\
\hline Cell suspension & & & High & & Yes & \\
\hline Spheroids & & & Moderate & & Yes & \\
\hline Tumor explants: & & & Low & & No & \\
\hline From primary cell lines & & & High & $\begin{array}{l}\text { Depends on } \\
\text { the cell line }\end{array}$ & & \\
\hline From patient tumors & & & Low & High & & \\
\hline \multicolumn{7}{|l|}{ Endogenous models } \\
\hline $\begin{array}{l}\text { Carcinogenically induced } \\
\text { (RT, OT, viral mutagenesis) }\end{array}$ & & & & & & No \\
\hline GEMMs & & & & & & No \\
\hline Transplantable GEMMs & & & & & & Yes \\
\hline
\end{tabular}

Note:-RT indicates radiation therapy; QT, chemotherapy.

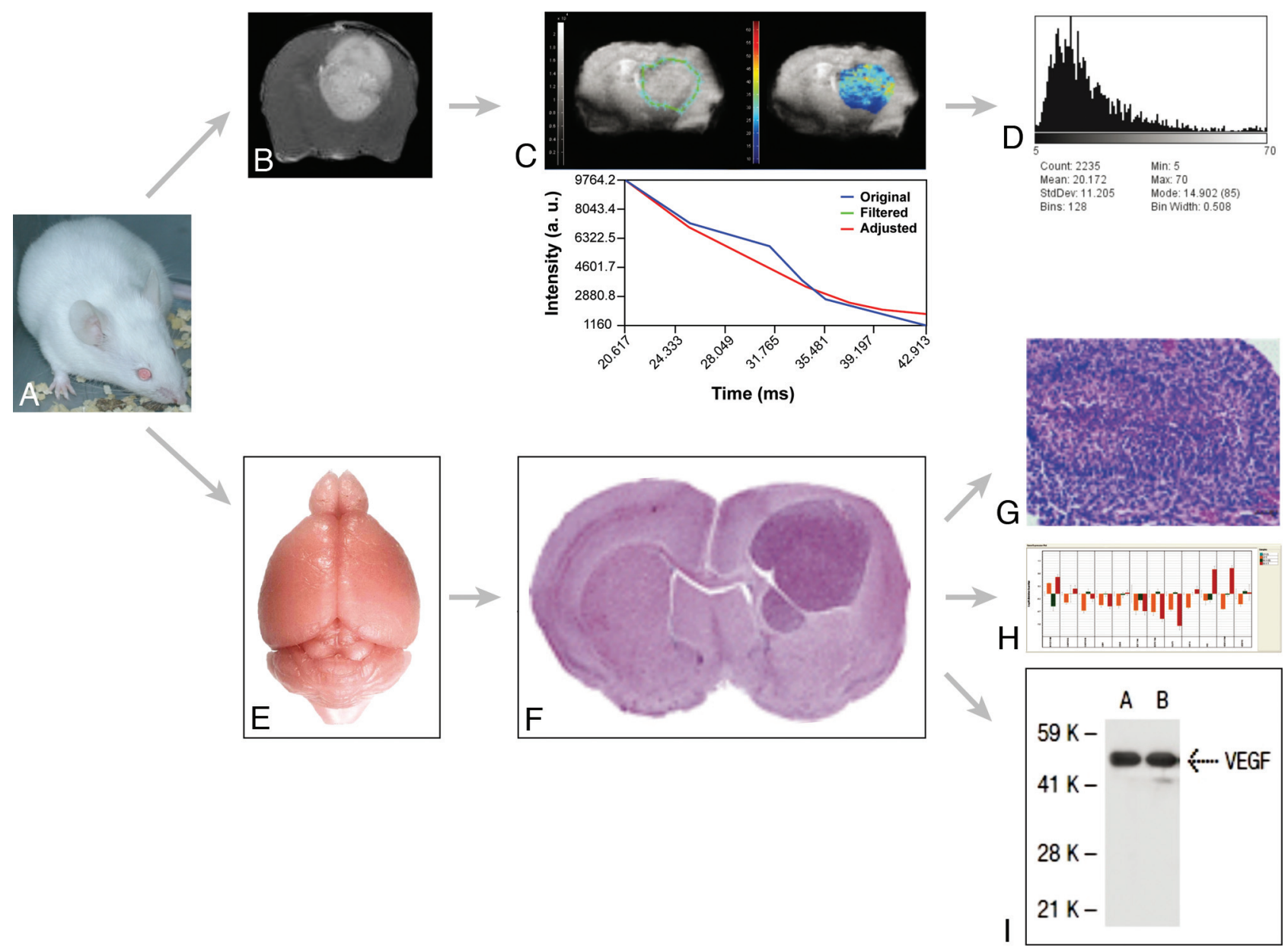

Fig 2. Flow chart for integrated MR imaging, histology, and genomic and proteomic approaches in models of HGG. $A$, Representative Swiss mouse. $B$, Gadolinium-enhanced axial T1-weighted image shows an implanted GI261 HGG. $C$, Parametric T2*map of the tumor in $B$. D. Parametric histogram of T2* values of $C$. E, Isolated normal brain from a representative Swiss mouse. F, Representative hematoxylin-eosin-stained section across a formalin-fixed paraffin-embedded mouse brain carrying an implanted $\mathrm{HGG}$. Reproduced from Lal et al ${ }^{17}$ with permission from the Journal of Neurosurgery Publishing Group. G. Microscopic view $(\times 40)$ of a fixed and stained section across an HGG (small black bar on the lower right corner represents $50 \mu \mathrm{m}$ ) shows pseudopalisading necrosis characteristic of HGG. Reproduced from Collier et al ${ }^{18}$ with permission from the American Association for Cancer Research. $\mathrm{H}$, Gene-expression profiling (RT qPCR results) from a representative GI261 HGG. I, Representative VEGF-A protein expression as detected by Western blot analysis. 

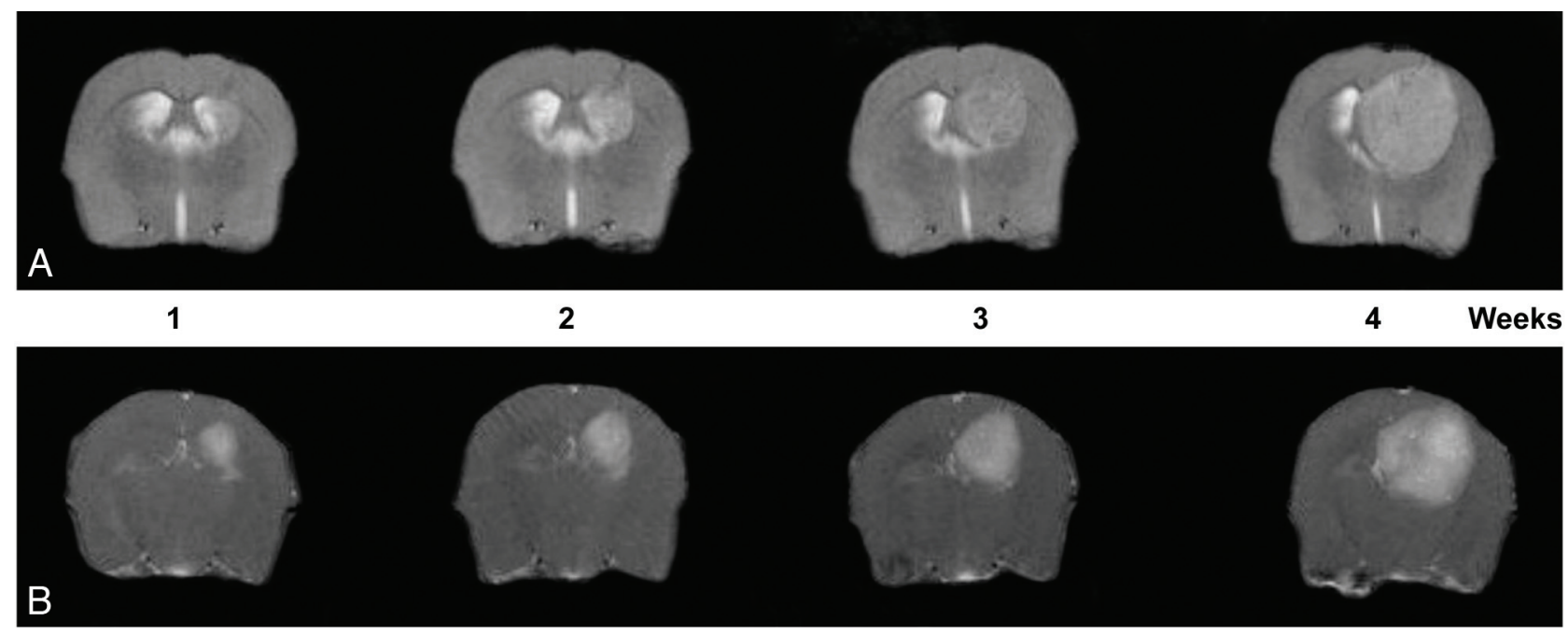

1

2

4

Weeks
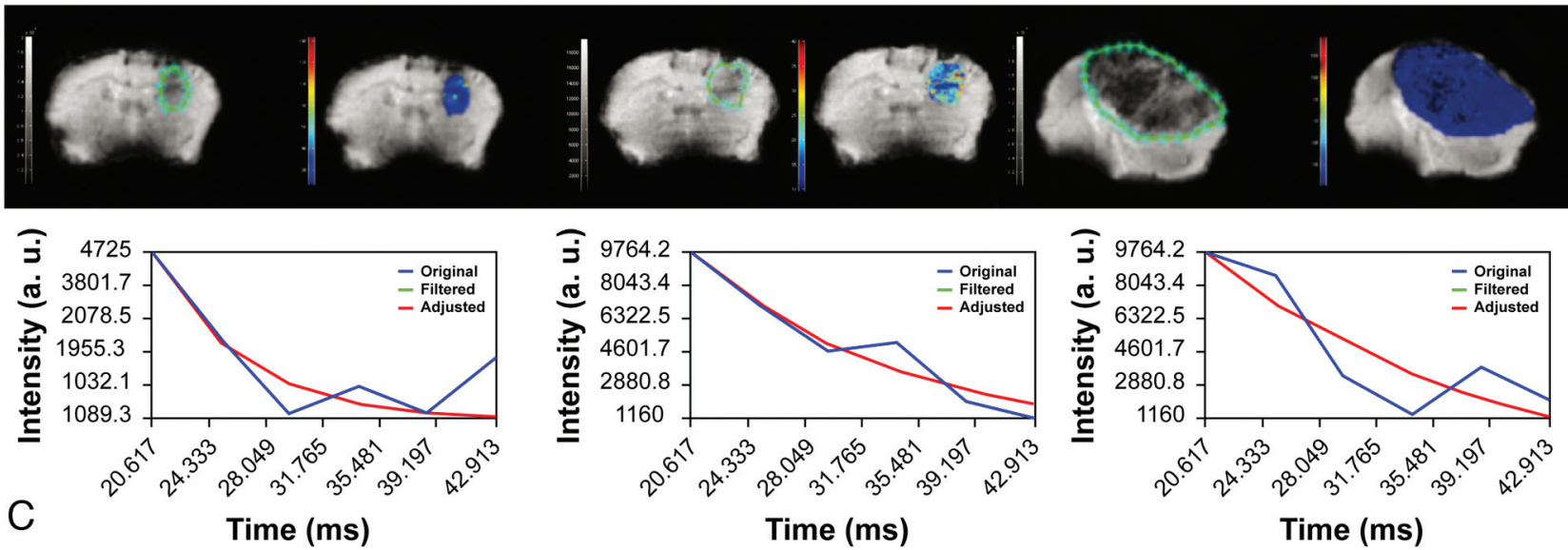

Time (ms)

Time (ms)
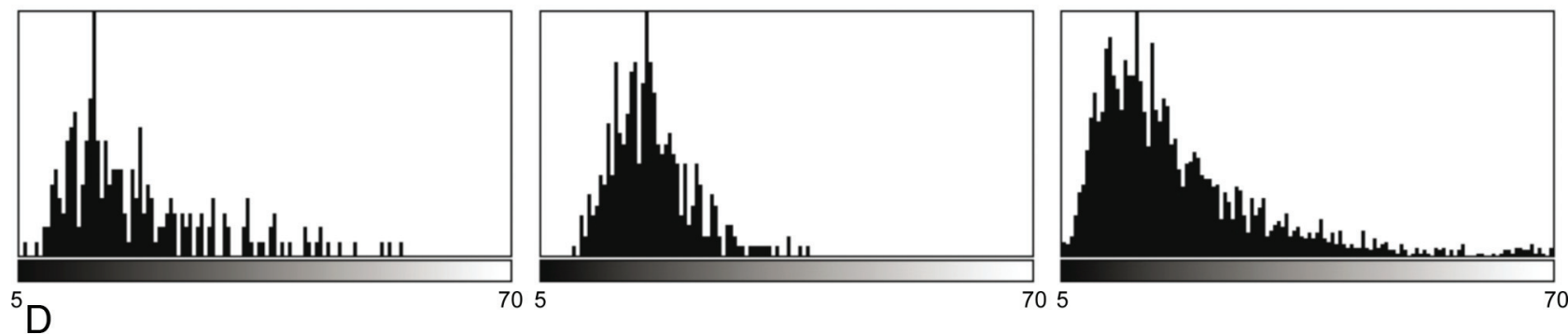

Fig 3. Serial axial T2-weighted $(A)$ and contrast-enhanced T1-weighted $(B)$ images of a GI261 C57/Bl6 mouse model of HGG, showing tumor growth and increased enhancement over a period of 4 weeks. $C$, ROls delineating the tumor (green limit) 1, 2, and 4 weeks after implantation; and nonlinear fitting (red) of signal intensity versus TE (blue) in representative pixel and parametric T2* maps depicting T2* values in the selected ROI. $D, T 2^{*}$ histograms nicely depict the temporal variation of T2* values with tumor progression modified by small differences in oxygen blood saturation reflected by the paramagnetic effect of deoxyhemoglobin.

coil and individual radio-frequency coils for each mouse allows smaller FOVs but requires appropriate shielding to isolate individual coils (Fig 6). Postprocessing is labor-intensive, requiring individual reconstruction of each separate dataset and FOV unwrapping due to phase-encoding aliasing in offcenter samples. Streamlining of scout views and simultaneous ROI selection for functional studies constitute additional problems. Acquisition of 3D isotropic images with retrospective ROI selection is preferred in these circumstances. The use of short TR sequences is also limited because to maintain image resolution, one must increase the duration of gradient pulses. To facilitate data analysis of multiple mouse MRI, coregistration, automated analysis, and image-segmentation packages are now available.

The use of MR spectroscopy in mice is even more challenging, requiring very small volumes of interest and custom high-order shim coils. Therefore, well-resolved spectra are difficult to obtain due to size and time constraints. Metabolic phenotyping can, however, be obtained ex vivo in pathologic specimens through the use of high-resolution magic angle spin spectroscopy. ${ }^{23}$

\section{MR Imaging Characterization of Mouse Models of HGG}

A MEDLINE search by using the search engines PubMed, Scopus, and Index Medicus and search terms "murine models of 

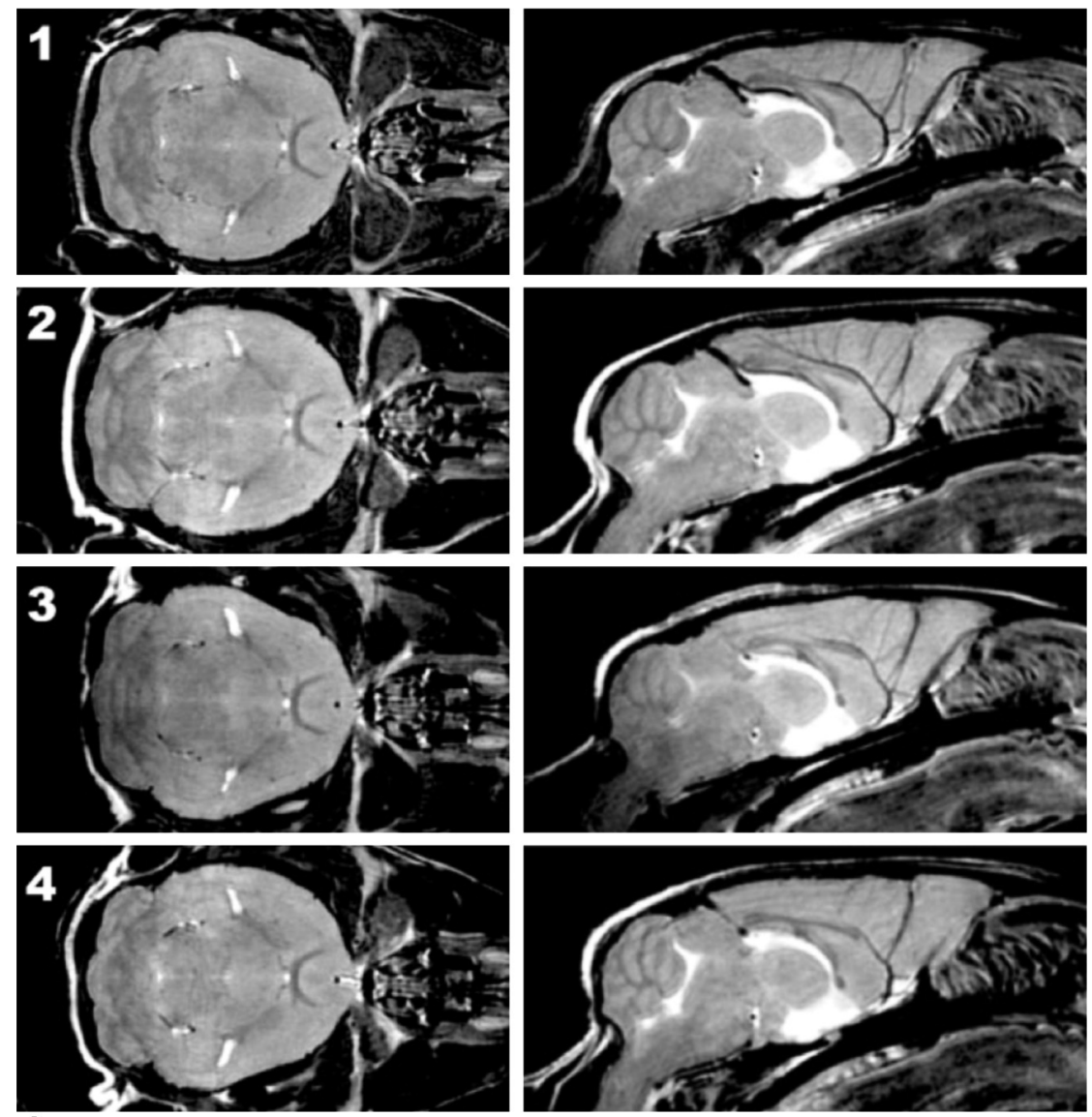

A
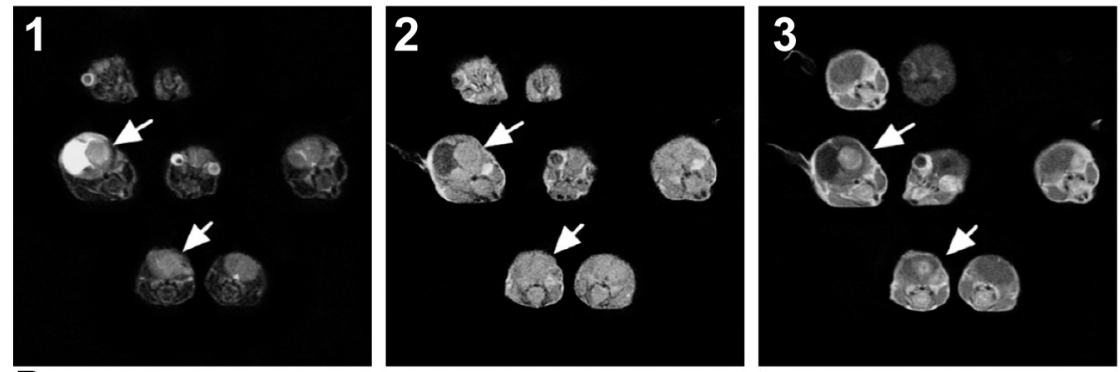

$\bar{B}$

Fig 4. A, Multiple mouse parallel imaging. Coronal (left) and sagittal (right) sections from FSE multiple mouse MRl images of 4 mice (numbered 1-4). Each 3D image has a 100- $\mu \mathrm{m}$ isotropic resolution. Scan duration was 2 hours 50 minutes. Reproduced from Nieman et al ${ }^{16}$ with permission from John Wiley and Sons. B, Multiple mouse MRI of GEMMs of HGG T2-weighted (1), T1-weighted (2), and gadolinium-enhanced T1-weighted (3) images, obtained on a 1.5T Signa clinical scanner (GE Healthcare, Milwaukee, Wisconsin) by using a common receiver coil, clearly demonstrates which mice developed brain tumors (white arrows) and differentiates brain tumors from hydrocephalus. Reproduced from Koutcher et al with the authors' permission. ${ }^{26}$

HGG," “neuroimaging features," "MR imaging features," and "MR imaging in preclinical trials" retrieved 50 studies, critically summarized below. MR imaging-based studies included conventional MR images either isolated or as part of a multimodality imaging approach, functional imaging (PWI, DWI and BOLD), and MR imaging applied in preclinical trials by using murine models of HGG.

\section{Conventional MR imaging}

In most of these studies, serial MR imaging was used to screen and monitor tumor growth, to characterize the imaging features of different models of HGG, and to correlate imaging phenotypes with tumor histology and gene expression to de- termine their predictive range (On-line Table 1). ${ }^{24-32}$ In murines, symptoms associated with tumor development are indistinguishable from those secondary to hydrocephalus. Therefore, imaging is mandatory to accurately screen for intracranial tumors.

The MR imaging features of the U87MG and U251 xenografts were characterized by Radaelli et al. ${ }^{25}$ These authors found a good correlation between the area of necrotic tumor and the intensity of gadolinium enhancement determined on serial MR imaging studies and quantitative scores of percentage necrosis and MVD found on histopathology. U251 tumors featured HGGs resembling the human counterpart (with illdefined infiltrating margins, necrosis, and surrounding 


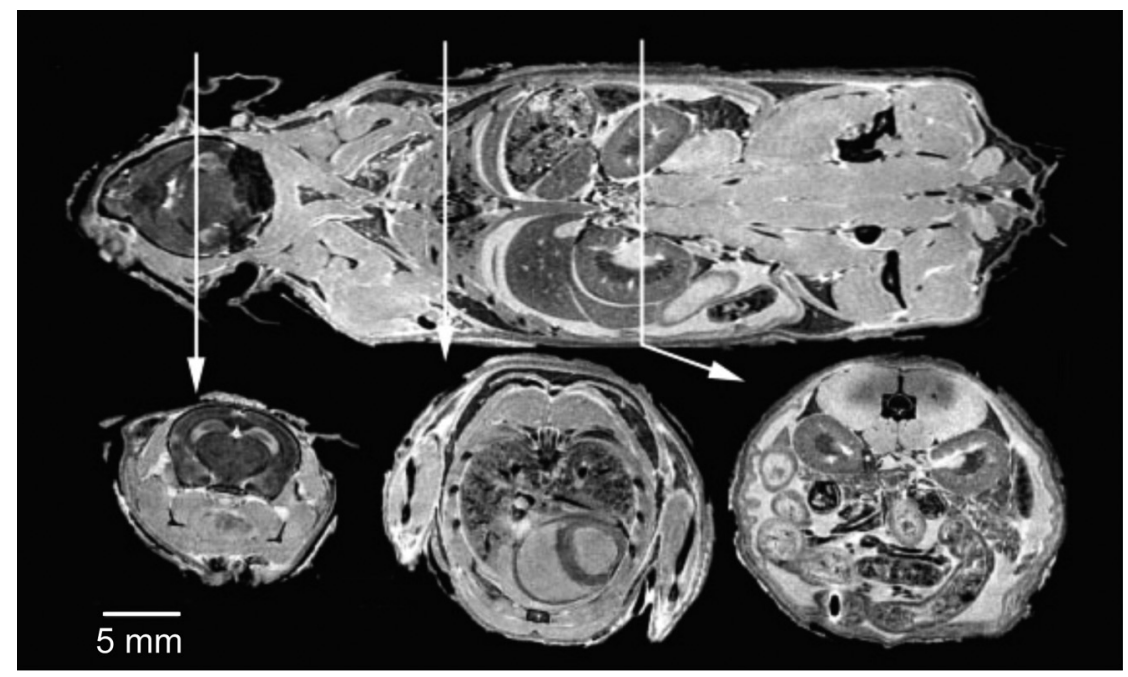

Fig 5. The visible mouse project (3D MR imaging of a whole fixed C57BI/6J mouse). Images acquired with an isotropic array with a $256 \times 256 \times 1024$ matrix and $110 \times 110 \times 110$ $\mu \mathrm{m}$ in-plane resolution. Coronal (top) and representative axial T1-weighted sections from the brain (left), thorax (center), and abdomen (right). Reproduced from Johnson et al ${ }^{21}$ with permission from the Radiologic Society of North America.

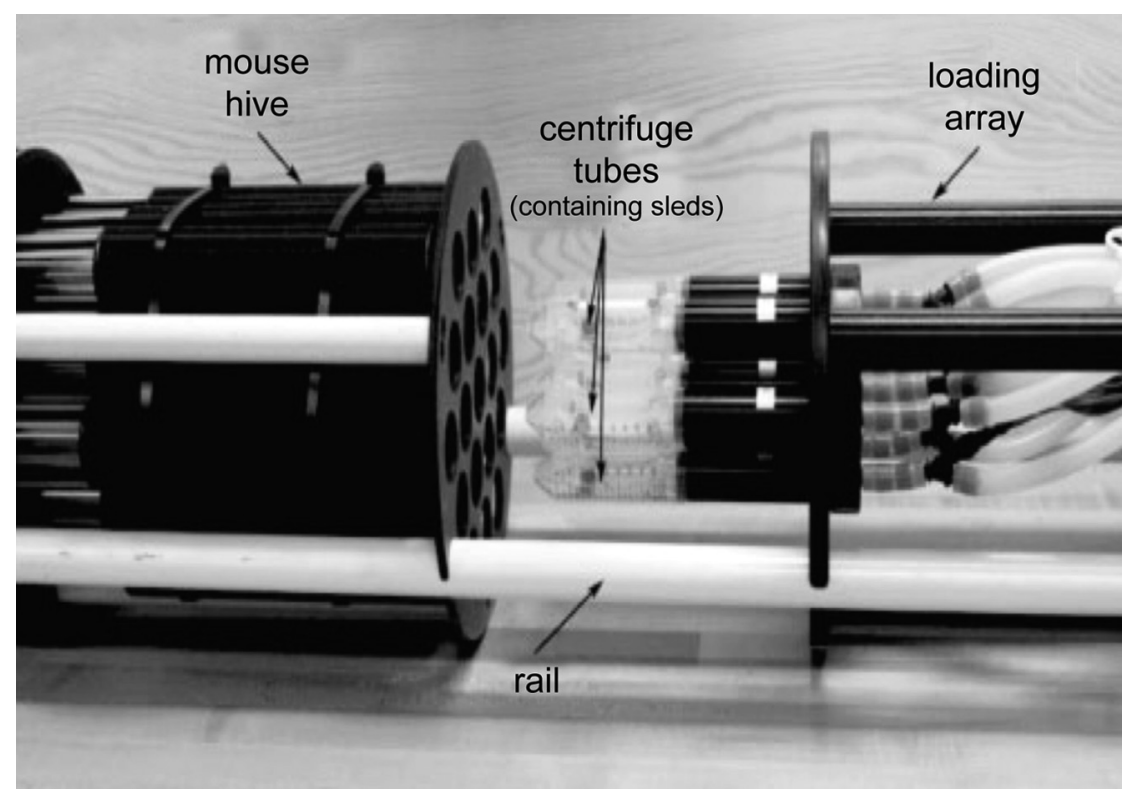

Fig 6. Multiple mouse MRI loading system described by Dazai et al. ${ }^{22}$ Mice loading array equipped with anesthesia and a mouse hive, which holds up to 19 receiver coils. Reproduced from Dazai et al ${ }^{22}$ with permission from John Wiley and Sons.

edema), whereas U87MG neoplasms were mostly rounded, well-demarcated from the surrounding brain, and closer to metastatic disease than to an aggressive primary brain tumor. However, strong gadolinium enhancement, featuring intense neoangiogenesis, makes this tumor model ideal to evaluate response to antiangiogenic drugs.

Koutcher et $\mathrm{a}^{26}$ used multiple mouse MRI for screening GEMMs of HGG by using a clinical 1.5T MR imaging scanner and an $86-\mathrm{cm}$ solenoid coil. MR imaging studies demonstrated a $20 \%-30 \%$ tumor take and a strong correlation between contrast enhancement and the presence of glomeruloid microvascular proliferation assessed on histology. Despite the low resolution, MR imaging was sensitive enough for tumor screening by using T1-weighted, T2-weighted, and contrastenhanced T1-weighted signal-intensity ratios between tumor and normal brain. Size-limit detection was $0.8 \mathrm{~mm}$ with 5 false-negatives below $1 \mathrm{~mm}$.

Various multimodality imaging studies included MR imaging to characterize HGG tumor models and to appraise treatment response.

The DBT orthotopic xenograft of HGG was featured by Jost et $\mathrm{al}^{24}$ by using a multimodality imaging approach which comprised MR imaging, PET, and optical imaging. Good correlation was found between tumor volumes determined on MR imaging studies and on the pathology specimen both in control and carmustine treatment groups given 1 or 2 carmustine doses, respectively. Tumor-growth curves and growth-rate exponents showed a 7-day delay of tumor growth burst in the single carmustine dose group and an 18-day delay on the 2-dose group, after which tu- 
mors returned to baseline growth rates approaching those of nontreated controls.

MR multispectral tissue segmentation was performed by Gordon et $\mathrm{al}^{27}$ in HJC-15c orthtotopic xenografts, by using a $1.5 \mathrm{~T}$ clinical scanner and a conventional wrist coil, to discriminate tumor boundaries and different tumor components. Automated multispectral segmentation analysis, by using input from 3 different 3D volumetric acquisitions (GRE, inversion recovery-GRE, and steady-state free precession) was able to discriminate tumor from normal brain, edema, and CSF and to separate different components of these heterogeneous tumors (necrotic from viable, enhancing from nonenhancing tumor) with higher reproducibility and better histologic correlation and in a shorter time than manual delineation based on visual assessment.

The influence of tumor models on the evaluation of drug efficacy on preclinical trials was demonstrated by Kemper et al. ${ }^{28}$ Several FVB xenografts known to grow behind an intact $\mathrm{BBB}$ (Mel 57, K173 Br2, and RG2) and to disrupt the BBB (U87MG and Mel57 transfected with VEGF-A) were imaged by $\mathrm{MR}$ and bioluminescence imaging. The intensity of contrast enhancement, measured on a dynamic GRE T1-weighted sequence after IV administration of gadolinium-DTPA, was positively correlated with MVD and immunohistochemistry for Ki67 (a proliferation marker) and glucose transporter 1 (a BBB marker absent in leaky vessels lacking a functional BBB). Not surprisingly, treatment efficacy of a 4-week course of oral temozolomide was higher in models with BBB disruption, featuring leakier vessels and more intense contrast enhancement (U87MG) than in models growing essentially behind an intact BBB with poor contrast enhancement. Whereas the former may overestimate, the latter may underestimate treatment response.

MR imaging coupled with diffuse optical fluorescence tomography was attempted by Davis et $\mathrm{al}^{29}$ to analyze tumor metabolism in a U-251 orthotopic xenograft. Authors were able to assess and visualize the metabolic activity of this tumor model in vivo by measuring and spatially localizing the fluorescence emitted by protoporphyrin IX (an endogenous fluorophore).

To validate bioluminescence imaging and MR imaging as quantitative tools to assess the efficacy of cytotoxic drugs, Rehemtulla et $\mathrm{al}^{30}$ quantified tumor volumes and log cell kill in a 9L orthotopic xenograft transfected with luciferase, both in control and carmustine treatment groups, by using histology as the criterion standard. Bioluminescence imaging slightly overestimated tumor size due to scatter of emitted photons, whereas MR imaging underestimated log cell kill because it measures both living and dead cells, cell debris, peritumoral edema, and infiltrating host cells as part of tumor volume, underscoring treatment effect. Besides the lower cost, shorter scanning times, high throughput, and minimal postprocessing requirements, bioluminescence imaging proved to be a better surrogate marker of active tumor volume because light output is only seen in metabolically active cells and does not account for necrotic tissue. Hence, bioluminescence imaging is a very good candidate for screening animals before more accurate, expensive, and time-consuming modalities, such as MR imaging or PET, are undertaken to provide spatial and or functional information.
Finally, Szentirmai et al, ${ }^{31}$ compared MR imaging and bioluminescence imaging in the evaluation of tumor-growth kinetics under antiangiogenic treatment in a U87MG luciferase transfected orthotopic xenograft. Because antiangiogenic treatments increase the fraction of hypoxic tumor cells, at least temporarily, and luciferase activity requires oxidation of the substrate (luciferin), bioluminescence imaging was shown to underestimate tumor volumes and overestimate treatment response.

\section{Functional MR Imaging}

Perfusion MR Imaging. Tumor blood volume is a very important biometric imaging target. Many studies have emerged in recent years attempting to validate MR imagingbased perfusion imaging as a biomarker of angiogenesis (Online Table 2). ${ }^{33-41} \mathrm{MR}$ imaging can provide information on tumor perfusion by tracking a bolus of an exogenously administered contrast agent or by tracking endogenous magnetically labeled flowing blood. The former includes DCE PWI, which measures the T1 shortening effect of the contrast agent, and DSC PWI, which measures the susceptibility effect of the contrast agent on $\mathrm{T} 2{ }^{\star}$ GRE images. The latter, known as arterial spin-labeling, measures signal-intensity differences between images obtained with and without a tagging pulse, which may be intermittent or continuous (pulsed arterial spin-labeling or continuous arterial spin-labeling). This technique is free of recirculation or residual tracer effects, does not require monitoring of tracer concentration time curves during the first passage, and, being completely noninvasive, may be repeated as often as required.

DCE-MR perfusion can be performed by using either small molecule contrast agents that escape the disrupted BBB in tumors and extravasate to the extracellular and extravascular space or macromolecular contrast agents that remain longer in the intravascular compartment. ${ }^{33,34}$ To minimize the effect of extravasated contrast in perfusion measurements, one must use an AIF to measure the rate of incoming blood in a nearby supplying artery or a pre-bolus injection. With a $3 \mathrm{D}$ equilibrium water-exchange model, it is possible to derive information on tumor blood volume; extravascular space vascular permeability, and $K^{\text {trans }} .33,34$

Perfusion parameters of the U87MG rat xenograft were characterized by Li et $\mathrm{al}^{33}$ by using DCE-PWI after the IV administration of $50 \mu \mathrm{mol} / \mathrm{Kg}$ of Omniscan (gadodiamide; Nycomed Amersham, Oslo, Norway). Using an AIF derived from the superior sagittal sinus (a technique previously validated in rats ${ }^{42}$ ), the authors were able to estimate CBV, CBF, $K^{\text {trans }}$, and vascular permeability from first-passage T1weighted DCE-PWI despite extensive extravasation of the contrast agent. However, in the absence of a criterion standard, the absolute accuracy of DCE-PWI is difficult to ascertain and is highly dependent on the accurate measurement of the AIF, quite difficult in the rat brain.

Adzamli et $\mathrm{al}^{34}$ compared different perfusion parameters and the delineation of abnormal vascular beds among 3 different albumin-binding contrast agents (MP-2269, MS-325, and gadoversetamide (OptiMARK; Mallinckrodt, St. Louis, Missouri) in the DBT mouse xenograft. Signal intensity was plotted with time and time-to-peak; plateau and washout time were compared with MVD and vascular morphology, assessed 
on histology. All contrast agents showed increased blood persistence compared with unbound agents (2.5 hours versus 10 minutes) and different kinetics of tumor uptake and clearance.

The effect of antiangiogenic drugs in different models of brain vascularization both in vitro and in vivo was assessed by Goldbrunner et al. ${ }^{35}$ These authors found increased expression of angiogenic molecules in spheroids compared with cell suspensions, which increased with the increasing size of spheroids reflecting the importance of the tumor-stroma cross-talk and the paracrine actions of angiogenic growth factors. The increased expression of integrin $\alpha 5 \beta 3$ in glioma endothelial cells can be imaged by tagging a monoclonal antibody against this protein with a paramagnetic contrast agent used as a marker for tumor angiogenesis (Gad-LM609-Ab anti- $\alpha 5 \beta 3) .{ }^{43}$

Perfusion imaging through endogenous labeling has also been explored in mouse models of HGG. Sun et $\mathrm{al}^{36}$ evaluated the performance of continuous arterial spin-labeling assessing perfusion of the U87MG mouse xenograft. Extracted imaging parameters, CBF indexes, and apparent $\mathrm{T} 1$ of the tumor core, tumor periphery, and normal brain positively correlated with radial histologic analysis of MVD. Progressive CBF decrease was noted from normal brain to tumor core and nicely correlated with the presence of nonfunctional morphologically abnormal intratumoral vessels with increased resistance and high permeability, which do not participate in effective microcirculation. The technique proved to be highly reproducible with low inter- and intraindividual variation.

The temporal correlation between DCE-PWI and tumor angiogenesis was appraised by Veeravagu et $\mathrm{al}^{37}$ and confronted with MVD and genetic expression of VEGF and angiotensin 1 and 2 assessed by real-time quantitative polymerase chain reaction. DCE-PWI studies were performed in the GL261 C57Bl/6 orthotopic xenograft after the administration of $0.1 \mathrm{mmol} / \mathrm{Kg}$ of Magnevist (gadopentetate dimeglumine; Schering, Berlin, Germany). A stepwise increase in time-topeak preceded tumor growth burst and coincided with an increase in MVD, vessel caliber, and increased expression of VEGF and angiotensin 2, responsible for endothelial sprouting and increased vascular leakiness. The decrease in signalintensity/time ratios immediately following exponential tumor growth concurred with vessel normalization and decreased leakiness and with the overexpression of angioten$\sin 1$, responsible for the maturation and stabilization of new blood vessels. Therefore, DCE-MR imaging reflects the functional and architectural status of growing blood vessels in vivo, being able to depict the angiogenic switch preceding rapid tumor growth and the normalization of the vascular support responsible for the infiltrating pattern of tumor growth behind an intact BBB.

Using DSC-PWI, Cha et $\mathrm{al}^{38}$ correlated rCBV with MVD and CD31 immunohistochemical staining and maximum vascular permeability with Evans blue extravasation in the Gl261 C57Bl/6 mouse xenograft. A single-section GRE T2* FLASH sequence after the IV administration of $0.1 \mathrm{mmol} / \mathrm{Kg}$ of Magnevist was performed, showing enhancement and increased rCBV at all stages of tumor progression, with regional and temporal differences. rCBV increased at the tumor periphery up to the first couple of weeks, reflecting the co-option and dilation of host vessels, decreased during the second and third weeks due to exponential tumor growth and host vessel insufficiency, and increased again on the fourth week secondary to frank tumor neovascularization at the tumor margins and surrounding centrally necrotic areas, lacking a functional $\mathrm{BBB}$.

Gossmann et $\mathrm{al}^{39}$ evaluated the role of DCE MR imaging as a surrogate marker of tumor response to anti-VEGF monoclonal antibody (A4.6.1) in a U87MG rat xenograft. High-resolution spoiled gradient-recalled echo T1-weighted images were obtained at different timings after a bolus injection of 0.1 $\mathrm{mmol} / \mathrm{Kg}$ of Magnevist, to extract enhancing tumor volumes and perfusion parameters. A statistically significant decrease in tumor volume, fractional plasma volume, and endothelial transfer coefficient was found between treated and control groups preceding any variation in tumor volume. Therefore, hemodynamic parameters may provide early monitoring of treatment efficacy and allow timely changes in treatment plan.

Muir et $\mathrm{al}^{40}$ quantified $\mathrm{CBF}$ in $\mathrm{C} 57 \mathrm{Bl} / 6$ mice by using a cardiac spin-labeling technique both in basal conditions and hypercapnia by placing the labeling coil in the heart instead of the neck, to avoid signal-intensity saturation. Animals were imaged with a head coil and a circular labeling coil at the heart position, in parallel and $2 \mathrm{~cm}$ apart. A GRE EPI sequence was used to measure absolute CBF values that were cross-validated through the use of microspheres, iodoantipyrine autoradiography, and laser speckle flowmetry.

\section{Diffusion MR Imaging}

DWI is sensitive to tissue structure at the cellular level, reflecting the dynamics of water molecules in the microscopic environment. Water diffusion inside a tumor is heterogeneous, accounting for regional differences in cellular density mainly due to tumor necrosis and apoptosis. This spatial heterogeneity can be easily depicted on ADC maps and histograms and may reflect regional differences in treatment response (Fig 7). ${ }^{44}$ Whereas a histogram shift to higher ADC values indicates a good response, a shift to lower values indicates poor or no response and precedes changes in tumor volume, allowing early assessment of treatment effectiveness. With high-field scanners, often used to image small animals, spin-echo-based DWI sequences have higher quality than EPI-based sequences because they are less prone to susceptibility artifacts. In most animal-dedicated scanners, high-resolution DWI data can be obtained within a reasonable timeframe by using spin-echo multisection sequences, isotropic diffusion, flow compensation, first-moment motion, and navigator echo correction. Until currently, most DWI studies were performed on rat models and only a few in mice (On-line Table 3). ${ }^{44-47}$

In a study by Moffat et $\mathrm{al},{ }^{44}$ DWI was used to evaluate treatment response to low and high doses of carmustine in a rat model of HGG. Parametric ADC maps and histograms predicted treatment response before any changes in tumor volume could be depicted. This early information can replace traditional therapeutic end points, allows timely secondary interventions, and, on clinical grounds, prevents the morbidity and cost of pursuing inefficient therapies.

Chenevert et $\mathrm{al}^{45}$ validated DWI as a surrogate marker of cell density after treatment with carmustine in a 9L rat model of HGG. Isotropic ADC maps and ADC pixel value histograms were obtained from whole tumor by manual delineation. Serial posttreatment ADC histograms showed increased diffu- 


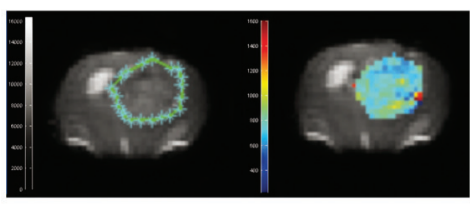

A

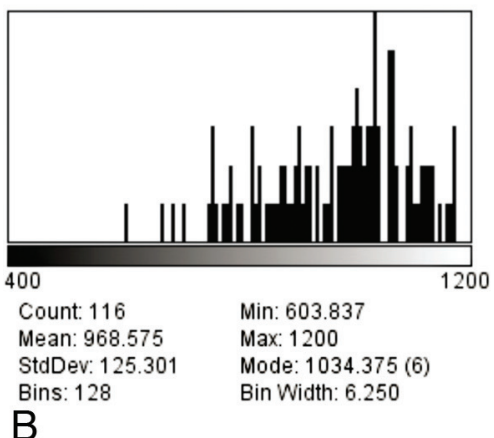

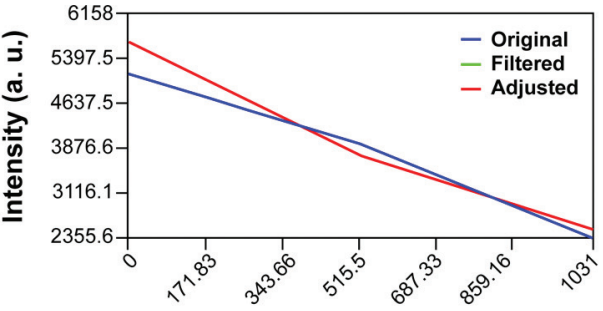

b $\left(\mathbf{s} / \mathrm{mm}^{\mathrm{s}}\right)$

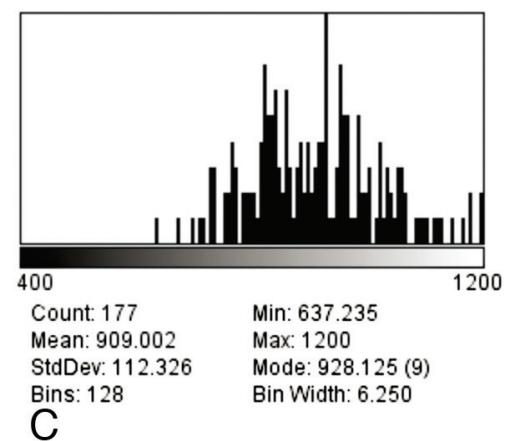

Fig 7. ADC imaging. A, Delineation of the ROI (left, green limits), intensity versus b value graph of a representative pixel (right), and a parametric color-coded map (center). Serial histograms over time on the second week $(B)$ and on the third week $(C)$ after tumor implantation depict a progressive leftward shift reflecting a decrease in ADC values secondary to increased tumor growth and cellularity.

sion peaking at day 8 after treatment, before any volume changes could be noted, and they correlated well with the course of cellular density over time, quantitatively assessed on histology.

The DWI features of the U87MG mouse xenograft were characterized by Sun et $\mathrm{al}^{46}$ by using a spin-echo DWI sequence with 2 b-values (100 and $750 \mathrm{~mm}^{2} / \mathrm{s}$ ). Radial assessment of mean ADC values from the tumor core to tumor periphery and surrounding normal-appearing brain were obtained, demonstrating regional differences within the tumor.

Fan et $\mathrm{al}^{47}$ correlated DWI and PWI parameters with cellular attenuation and MVD respectively, on a rat model of C6 HGG. These authors used an EPI-DWI sequence with $2 \mathrm{~b}$-values $\left(0\right.$ and $\left.1000 \mathrm{~mm}^{2} / \mathrm{s}\right)$ on a $1.5 \mathrm{~T}$ clinical scanner. ADC values averaged from 3 to 5 different ROIs were compared with histologic assessment of cell density. A gradual decrease of mean ADC values was seen during a 4-week period, but correlation with cellular density was only moderate $(r=0.682)$ in this study. The authors found, however, that peritumoral ADC values could be used to discriminate tumor infiltration from edema.

\section{Hypoxia Imaging (BOLD, T2*-Weighted)}

Tumor oxygenation is of utmost importance because oxygen regulates gene expression through the activation and inhibition of several transcription factors controlling genetic modules of angiogenesis, metabolism, migration, invasion, and proliferation. ${ }^{48}$ It has long been recognized that the oxygenation status of a tumor predicts its response to radiation therapy and correlates with regional tumor control. ${ }^{49}$ Therefore, noninvasive determination of regional hypoxic fractions of a tumor became a major imaging goal. This information can be used to identify patients most likely to benefit from radiosensitizers and hypoxia selective drugs. ${ }^{49}$

The criterion standard method to evaluate tumor oxygen- ation is the use of polarographic oxygen microelectrodes introduced within the tumor. Besides being invasive, this technique measures the oxygen tension in limited areas of the tumor and does not account for regional heterogeneity.

Several imaging techniques have attempted to measure tumor hypoxia either directly or indirectly. The most promising has been PET imaging by using the ${ }^{18}$ fluorine 18 -labeled misonidazole radiotracer or under ${ }^{15} \mathrm{O}$ inhalation. Nevertheless, fluorine 18-labeled misonidazole shows significant binding only when oxygen tension is very low.

${ }^{1} \mathrm{H}$ and ${ }^{31} \mathrm{P} \mathrm{MR}$ spectroscopy provide indirect measures of oxygen tension by evaluating lactate/creatine, monoester/ ATP, and inorganic phosphorous/ATP ratios, respectively, which reflect the rate of anaerobic glycolysis under hypoxia: the lower the oxygen tension, the higher these ratios.

PWI, by providing information on MVD and vascular permeability, can also be regarded as an indirect measure of the hypoxic fraction of a tumor, though it does not account for either the rate of oxygen extraction or for nonfunctional microvessels that do not participate in effective tumor perfusion.

Another promising MR imaging technique is the use of BOLD ( $2^{*}$-weighted) imaging. $\mathrm{T} 2^{*}$ is modified by small differences in oxygen blood saturation through the paramagnetic effect of deoxyhemoglobin. Although the BOLD effect is also influenced by blood flow and blood volume, Goldbrunner et $\mathrm{al}^{35}$ demonstrated the presence of perfusion/oxygenation mismatches by using both techniques $\left(\mathrm{rCBF} / \mathrm{T} 2^{\star}\right)$.

\section{Applications in Preclinical Trials}

Robust model systems are mandatory for an efficient and rapid screening of drugs or combinations of drugs that target specific oncogenic pathways. In fact, the quality of preclinical trials depends on appropriate model selection and on the identification of relevant biomarkers of drug efficacy. ${ }^{2,3,5,7}$ Whereas in vitro systems, by using cell lines, are easier to con- 
trol, only in vivo systems can adequately recapitulate the cellular heterogeneity of tumors and the complexity of tumorhost interactions.

The ideal mouse model for drug development should provide a faithful recapitulation of the genetic and molecular features of the native tumor, be easy to use, have a high penetrance and a short and predictable latency, and allow timely evaluation of treatment effect (On-line Table 4). ${ }^{1,3,19,32,39,44,45,50-56}$

It is not unusual that drugs that appear very promising in preclinical trials have disappointing clinical results. ${ }^{2,14} \mathrm{~A}$ recent review by Amarasingh et $\mathrm{al}^{51}$ assessed the impact of chemotherapy drug research in neuro-oncology. These authors performed a stratified meta-analysis on the efficacy of nitrosureas (carmustine and lomustine) in animal models of HGG to generate global efficacy estimates. Problems were manifold: Most studies enrolled a limited number of animals and methodologies varied widely from one study to another in terms of animal model, treatment timing, dose scheduling, and administration route, disturbing global analyses. Several biases in animal experiments, including randomization, allocation, concealment, and outcome assessment, may be responsible for the overall increased efficacy of drugs in animal studies. Other shortcomings included neuropathologic differences with human neoplasm and a wide variation in the biologic behavior and genetic profiling of different glioma cell lines.

Many studies assessed the efficacy of antiangiogenic agents in animal models of HGG. ${ }^{19,39,44-46,51-56}$ Moffat et $\mathrm{al}^{52}$ compared tumor growth, perfusion parameters, and chemotherapy effectiveness in wild type, VEGF-A overexpressing, and VEGF-A underexpressing 9L gliosarcoma rat xenografts. Perfusion parameters, $\mathrm{CBV}$ and $\mathrm{CBF}$, were quantified both by DSC-PWI and continuous arterial spin-labeling and were confronted with MVD and immunohistochemical and genetic analysis by real-time quantitative polymerase chain reaction. Compared with wild type, VEGF-A overexpressing tumors showed an increased growth rate; a decreased mean survival time; and an increased CBF, CBV, and MVD. VEGF-A underexpressing tumors showed a decreased growth rate and an increased mean survival but increased CBF, CBV, and MVD due to the compensatory overexpression of VEFG-D. This study demonstrated that suppression of an angiogenic pathway can be counteracted by the compensatory overexpression of another, accounting for the adaptive ability of neoplasms. Antiangiogenic drugs decrease CBV by suppressing new blood vessel formation and decreasing vascular permeability, but they cause a slight increase in CBF due to the normalization of pre-existing blood vessels, which ameliorates tumor oxygenation and blood delivery to tumor cells, supporting the increased efficacy of cytotoxic therapies. Therefore, CBV and CBF constitute different and independent biomarkers of perfusion, providing complementary information about tumor vascularization.

De Groot et $\mathrm{al}^{19}$ assessed the imaging and biologic features of HGG after antiangiogenic treatment with bevacizumab (monoclonal antibody against VEGF receptor), both in humans and in a highly angiogenic poorly infiltrative U87 mouse xenograft. After a 4- to 6-week treatment course, MR imaging showed a shift to a highly infiltrating poorly angiogenic phenotype in humans that was accompanied by the overexpres- sion of insulin growth factor-binding protein 2, membranebound MMP2, carbonic anhydrase 9, and coagulation factor 8 and by tumor resistance to any subsequent treatments. Histologic analysis of human tumor biopsies has shown decreased MVD, microvessel normalization, and invasion of the neuropil, and that of mice tumors showed very invasive borders, perivascular and subpial invasion, and recovery of BBB integrity. Genetic analysis underscored insulin growth factorbinding protein 2 and MMP2 as the main mediators of this phenotypic shift in tumors evading chronic antiangiogenic treatment.

To evaluate the impact of bevacizumab on the antitumor efficacy of temozolomide, Mathieu et $\mathrm{al}^{53}$ treated 4 different cell lines and a mouse model of HGG with bevacizumab, temozolomide, and the combination of both. The combined treatment group showed increased survival and an antiangiogenic effect of temozolomide operating through a mechanism different from that of bevacizumab (overexpression of galectin 1).

McConville et $\mathrm{al}^{54}$ used the Ntv-a GEMM of HGG to appraise the role of conventional MR imaging and DWI in glioma grading and in early prediction of treatment response to temozolomide. MR imaging was able to predict tumor grading among the heterogeneous group of high- and low-grade gliomas generated by this model, treatment response, and survival. DWI, in particular, was able to monitor therapy-induced cell kill: Changes in net ADC values predicted volumetric change as early as $4-5$ days after the beginning of treatment.

Breton et $\mathrm{al}^{55}$ monitored the effect of suicide gene therapy in the U87MG mouse model by using manual segmentation of $\mathrm{T} 1$ and T2 tumor volumes on serial MR imaging studies performed on a low-field $0.1 \mathrm{~T}$ scanner. The authors determined tumor doubling times before and after treatment with suicide genes (injected locally) and the prodrug 5-fluorocytosine, proving the efficacy of this approach.

The efficacy of an oncolytic viral treatment for HGG by using glioblastoma multiforme spheroid xenografts orthotopically implanted in rats was assessed by Huszthy et al. ${ }^{56}$ After MR imaging identification, tumors were stereotactically injected with the oncolytic vector. Tumor growth was followed by contrast-enhanced MR imaging and was correlated with histologic analysis to assess proliferation, apoptosis, angiogenesis, and infiltration by inflammatory cells (CD68-positive microglia). Infected tumors showed a decreased proliferation index, MVD, and vessel area fraction and an increased apoptosis and microglia in central tumor areas.

\section{Current Developments/Molecular Imaging}

New imaging applications are quickly emerging and are being applied to animal models, mostly to provide molecular information bridging the gap between neuroimaging and neurobiology. ${ }^{57,58}$ The increasing use of biologic treatment regimens is driving the need to identify the biologically active target tissue. Oncogenetic changes serve as specific targets for tailored therapies and can be depicted by noninvasive imaging. Molecular imaging provides a noninvasive visual representation of biologic processes at the cellular and molecular levels in the $3 \mathrm{D}$ space, without the associated morbidity and time consumed on tissue sampling. ${ }^{58}$ 
Transfection techniques enable the visualization of transgene expression by coupling, in the same amplicon, 1 or several reporter genes that can be visualized by different imaging techniques with a gene of interest or via promoter-based coexpression. ${ }^{59}$ Increased expression of transferring receptor at the cell membrane increases iron binding $>500 \%$ after the administration of iron-based contrast agents. ${ }^{60}$ This simple system allows the visualization of cells/tissues expressing this transgene on $\mathrm{MR}$ imaging, through a $\mathrm{T} 2^{*}$ signal-intensity drop, which then functions as a surrogate marker of transgene expression. ${ }^{60}$

Fluorescent imaging of signaling transduction pathways has also been achieved. One of the best known systems in neuro-oncology is the dual reporter gene cis-p53/tirosine $k i$ nase- green fluorescent protein, reflecting the transcriptional activity of the tumor-suppressor gene $p 53$, induced by DNA damage.

Radiolabeling can also be used to approach transgene expression and monitor gene therapy by using SPECT or PET. The most promising radiotracers in neuro-oncology include fluorine-labeled misonidazole, fluorodeoxythymidine, and annexin $\mathrm{V}$ to image hypoxia, proliferation, and apoptosis, respectively. Under extensive research are the ${ }^{11} \mathrm{C}$-radiolabeled antisense oligonucleotide for the visualization of the glial fibrillary acidic protein messenger ribonucleic acid expression and ${ }^{123}$ I VEGF to visualize VEGF receptor. ${ }^{59}$

Near-infrared optic imaging has a vast potential for the visualization of biologic processes. The main shortcoming is the narrow penetrance of visible light, limiting its use to superficial neoplasms. In orthotopic models of brain tumors, near-infrared optic imaging is invasive, requiring a "brain window" to visualize the emitted light. However, coupled with fluorescence tomography, it not only allows the visualization of deep-seated tumors but also provides spatial localization. Using diffuse optic tomography and a brain window, Saxena et $\mathrm{al}^{61}$ identified the hypoxic fraction of a U87MG mouse model of HGG by using a green fluorescent protein reporter gene under the hypoxia responsive element promoter.

Tumor metabolomics is another promising research field in animal models of HGG. Using MR spectroscopy and mass spectrometry to explore metabolite profiling in brain tumors, Griffin et $\mathrm{al}^{62}$ found a good correlation between metabolic phenotypes and tumor type, proliferation index, metabolic activity, and cell death. Tumor metabolism can be approached in vivo by using MR spectroscopy (which provides additional spatial information and a metabolic map of the entire tumor) or ex vivo in tissue samples by high-resolution magic angle spin spectroscopy or in solution-state tumor extracts by mass spectrometry. Currently the use of ${ }^{1} \mathrm{H},{ }^{31} \mathrm{P}$, and ${ }^{13} \mathrm{C}$ MR spectroscopy pictures a wide range of metabolites belonging to different classes of compounds. An important application is the discrimination of cell death mechanisms through metabolic lipid profiling by ${ }^{1} \mathrm{H}-\mathrm{MR}$ spectroscopy, whereas the presence of saturated lipids indicates cell death by necrosis, and polyunsaturated fatty acids indicate cell death by apoptosis. ${ }^{13} \mathrm{C}$ spectra estimates glycolytic and oxidative metabolism and can depict metabolic shifts from oxidative to anaerobic and glucose carbon shunting surgery to anabolic pathways. Hyperpolarized ${ }^{13} \mathrm{C}$-containing substrates can be used to monitor oxidative and anaerobic metabolism in vivo without the use of radioactivity, and they are more sensitive than PET.

Multimodality imaging systems provide complementary information on a single examination. PET/CT already in clinical use and PET-MR imaging, on its way, combines molecular and functional information with high-resolution anatomic imaging. Trimodal (functional, metabolic, and anatomic) imaging data can be integrated to outline tumor target and functional risk areas. Radiolabeled, fluorescent, and paramagnetic probes can be used together to directly visualize endogenous proteins by PET, optical imaging, and MR imaging, respectively.

\section{Outlook}

A wide range of research and preclinical therapeutic trials on neurologic disorders rely on animal, mostly murine, studies. Progressive refinements of MR imaging hardware and software made neuroimaging of small animals, such as mice, increasingly powerful. Currently, all available functional MR imaging studies can be applied to murines, dramatically improving the value of animal-based preclinical trials and providing longitudinal noninvasive surrogate markers of biologic targeted end points for specific drugs. This change led to remarkable savings both in time and animal burden. Neuroimaging research in the areas of molecular imaging and genetically driven reporter probes is also being increasingly applied in murines. Major advances in the field of genetic engineering also led to the emergence of GEMMs, which are now invaluable for the understanding of oncogenic pathways driving tumor initiation and maintenance as well as the development of drugs targeting these pathways. Finally, the possibility of studying actual patient neoplasms by direct implantation of tumor explants, spheroids, or derived primary cell line suspensions in mice widens the use of animals to support personalized treatment approaches. On these grounds, it is important that radiologists become aware of this expanding field of research which can determine, in the future, the accurate biologic characterization of individual tumors and the concomitant development of a variety of molecularly targeted and cellbased therapies.

Disclosures: José Bravo-Marques—UNRELATED: Consultancy: Roche Framacêutica, Portugal, Travel/Accommodations/Meeting Expenses Unrelated to Activities Listed: Roche Framacêutica, Portugal. Sebastian Cerdan—RELATED: Grant. Ministerio de Ciencia e Innovación, UNRELATED: Employment: Consejo Superior de Investigaciones Científicas.

\section{References}

1. Barth RF, Kaur B. Rat brain tumor models in experimental neuro-oncology: the C6, 9L, T9, RG2, F98, BT4C, RT-2 and CNS-1 gliomas. J Neurooncol 2009;94:299-312

2. Gutmann DH, Hunter-Schaedle K, Shannon KM. Harnessing preclinical mouse models to inform human clinical cancer trials. J Clin Invest 2006;116:847-52

3. Fomchenko EI, Holland EC. Mouse models of brain tumors and their applications in preclinical trials. Clin Cancer Res 2006;12:5288-97

4. Miura FK, Alves MJ, Rocha MC, et al. Xenograft transplantation of human malignant astrocytoma cells into immunodeficient rats: an experimental model of glioblastoma. Clinics (Sao Paulo) 2010;65:305-09

5. Taillandier L, Antunes L, Angioi-Duprez K.S. Models for neuro-oncological preclinical studies: solid orthotopic and heterotopic grafts of human gliomas into nude mice. J Neurosci Methods 2003;125:147-57

6. Wang J, Miletic H, Sakariassen P $\varnothing$, et al., A reproducible brain tumour model established from human glioblastoma biopsies. BMC Cancer 2009;9:465

7. Holland EC. Brain tumor animal models: importance and progress. Curr Opin Oncol 2001;13:143-47 
8. Weiss WA, Israel M, Cobbs C, et al. Neuropathology of genetically engineered mice: consensus report and recommendations from an international forum. Oncogene 2002;21:453-63

9. Xie Q, Thompson R, Hardy K, et al. A highly invasive human glioblastoma pre-clinical model for testing therapeutics. J Transl Med 2008;6:77

10. Aldape K, Colman H, James C. Models of malignant glioma. Drug Discovery Today: Disease Models 2996;3:191-96

11. Smilowitz HM, Weissenberger J, Weis J, et al. Orthotopic transplantation of v-src-expressing glioma cell lines into immunocompetent mice: establishment of a new transplantable in vivo model for malignant glioma. J Neurosurg 2007;106:652-59

12. Huse JT, Holland EC. Genetically engineered mouse models of brain cancer and the promise of preclinical testing. Brain Pathol 2009;19:132-43

13. Marumoto T, Tashiro A, Friedmann-Morvinski D, et al. Development of a novel mouse glioma model using lentiviral vectors. Nat Med 2009;15:110-06. Epub 2009 Jan 4

14. Masdeu JC, Bakshi R. Neuroimaging: anything to do with neurotherapeutics? NeuroRx 2005;2:163-66

15. Lyons SK. Advances in imaging mouse tumour models in vivo. J Pathol 2005;205:194-205

16. Nieman BJ, Bock NA, Bishop J, et al. Magnetic resonance imaging for detection and analysis of mouse phenotypes. NMR Biomed 2005;18:447-68

17. Lal S, Lacroix M, Tofilon $P$, et al. An implantable guide-screw system for brain tumor studies in small animals. J Neurosurg 2000;92:326-333

18. Collier LS, Adams DJ, Hackett CS, et al. Whole-body sleeping beauty mutagenesis can cause penetrant leukemia/lymphoma and rare high-grade glioma without associated embryonic lethality. Cancer Res 2009;69:8429-37. Epub 2009 Oct 20

19. de Groot JF, Fuller G, Kumar AJ, et al. Tumor invasion after treatment of glioblastoma with bevacizumab: radiographic and pathologic correlation in humans and mice. Neuro Oncol 2010;12:233-42. Epub 2010 Jan 6

20. Radaelli E, Ceruti R, Patton V, et al. Immunohistopathological and neuroimaging characterization of murine orthotopic xenograft models of glioblastoma multiforme recapitulating the most salient features of human disease. Histol Histopathol 2009;24:879-91

21. Johnson GA, Cofer GP, Gewalt SL, et al. Morphologic phenotyping with MR microscopy: the visible mouse. Radiology 2002;222:789-93

22. Dazai J, Bock NA, Nieman BJ, et al. Multiple mouse biological loading and monitoring system for MRI. Magn Reson Med 2004;52:709-15

23. Righi V, Roda JM, Paz J, et al. 1H HR-MAS and genomic analysis of human tumor biopsies discriminate between high and low grade astrocytomas. NMR Biomed 2009;22:629-37

24. Jost SC, Wanebo JE, Song SK, et al. In vivo imaging in a murine model of glioblastoma. Neurosurgery 2007;60: 360-70, discussion 370-71

25. Radaelli E, Ceruti R, Patton V, et al. Immunohistopathological and neuroimaging characterization of murine orthotopic xenograft models of glioblastoma multiforme recapitulating the most salient features of human disease. Histol Histopathol 2009;24: 879-91

26. Koutcher JA, Hu X, Xu S, et al. MRI of mouse models for gliomas shows similarities to humans and can be used to identify mice for preclinical trials. $\mathrm{Neo}$ plasia 2002;4:480-85

27. Gordon J, Mohamed F, Vinitski S, et al. Utilization of experimental animal model for correlative multispectral MRI and pathological analysis of brain tumors. Magn Reson Imaging 1999;17:1495-502

28. Kemper EM, Leenders W, Küsters B, et al. Development of luciferase tagged brain tumour models in mice for chemotherapy intervention studies. Eur J Cancer 2006;2:3294-303. Epub 2006 Oct 5

29. Davis SC, Pogue BW, Springett R, et al. Magnetic resonance-coupled fluorescence tomography scanner for molecular imaging of tissue. Rev Sci Instrum 2008;79:064302

30. Rehemtulla A, Stegman LD, Cardozo SJ, et al. Rapid and quantitative assessment of cancer treatment response using in vivo bioluminescence imaging. Neoplasia 2000;2:491-95

31. Szentirmai $\mathrm{O}, \mathrm{Baker} \mathrm{CH}, \mathrm{Lin} \mathrm{N}$, et al. Noninvasive bioluminescence imaging of luciferase expressing intracranial U87 xenografts: correlation with magnetic resonance imaging determined tumor volume and longitudinal use in assessing tumor growth and antiangiogenic treatment effect. Neurosurgery 2006;58: 365-72, discussion 365-72

32. Bryant MJ, Chuah TL, Luff J, et al. A novel rat model for glioblastoma multiforme using a bioluminescent F98 cell line. J Clin Neurosci 2008;15:545-51

33. Li X, Rooney WD, Várallyay CG, et al. Dynamic-contrast-enhanced-MRI with extravasating contrast reagent: rat cerebral glioma blood volume determination. J Magn Reson 2010;206:190-99. Epub 2010 Jul 31

34. Adzamli K, Yablonskiy DA, Chicoine MR, et al. Albumin-binding MR blood pool agents as MRI contrast agents in an intracranial mouse glioma model. Magn Reson Med 2003;49: 586-90

35. Goldbrunner RH, Wagner S, Roosen K, et al. Models for assessment of angiogenesis in gliomas. J Neurooncol 2000;50:53-62
36. Sun Y, Schmidt NO, Schmidt K, et al. Perfusion MRI of U87 brain tumors in a mouse model. Magn Reson Med 2004;51: 893-99

37. Veeravagu A, Hou LC, Hsu AR, et al. The temporal correlation of dynamic contrast-enhanced magnetic resonance imaging with tumor angiogenesis in a murine glioblastoma model. Neurol Res 2008;30: 952-59. Epub 2008 Jul 25

38. Cha S, Johnson G, Wadghiri YZ, et al. Dynamic, contrast-enhanced perfusion MRI in mouse gliomas: correlation with histopathology. Magn Reson Med 2003;49:848-55

39. Gossmann A, Helbich TH, Kuriyama N, et al. Dynamic contrast-enhanced magnetic resonance imaging as a surrogate marker of tumor response to antiangiogenic therapy in a xenograft model of glioblastoma multiforme. J Magn Reson Imaging 2002;15:233-40

40. Muir ER, Shen Q, Duong TQ. Cerebral blood flow MRI in mice using the cardiac-spin-labeling technique. Magn Reson Med 2008;60:744-48

41. Wong ET, Brem S. Antiangiogenesis treatment for glioblastoma multiforme: challenges and opportunities. J Natl Compr Canc Netw 2008;6:515-22

42. Nagaraja TN, Ewing JR, Karki K, et al. MRI and quantitative autoradiographic studies following bolus injections of unlabeled and (14)C-labeled gadolinium-diethylenetriaminepentaacetic acid in a rat model of stroke yield similar distribution volumes and blood-to-brain influx rate constants. NMR Biomed 2011;24:547-58. Epub 2010 Dec 12

43. Sipkins DA, Cheresh DA, Kazemi MR. Detection of tumor angiogenesis in vivo by alphaVbeta3-targeted magnetic resonance imaging. Nat $\mathrm{Med}$ 1998;4:623-26

44. Moffat BA, Hall DE, Stojanovska J, et al., Diffusion imaging for evaluation of tumor therapies in preclinical animal models. MAGMA 2004;17:249-59. Epub 2004 Dec 1

45. Chenevert TL, Stegman LD, Taylor JM, et al. Diffusion magnetic resonance imaging: an early surrogate marker of therapeutic efficacy in brain tumors. J Natl Cancer Inst 2000;92:2029-36

46. Sun Y, Mulkern RV, Schmidt K, et al. Quantification of water diffusion and relaxation times of human U87 tumors in a mouse model. NMR Biomed 2004;17:399-404

47. Fan $G$, Zang $P$, Jing F, et al. Usefulness of diffusion/perfusion-weighted MRI in rat gliomas: correlation with histopathology. Acad Radiol 2005;12:640-51

48. Pacheco-Torres J, López-Larrubia P, Ballesteros P, et al. Imaging tumor hypoxia by magnetic resonance methods. NMR Biomed 2011;24:1-16. Epub 2010 Dec 9

49. Yetkin FZ, Mendelsohn D. Hypoxia imaging in brain tumors. Neuroimaging Clin N Am 2002;12:537-52

50. de Vries NA, Beijnen JH, van Tellingen O. High-grade glioma mouse models and their applicability for preclinical testing. Cancer Treat Rev 2009;35:714-23

51. Amarasingh S, Macleod MR, Whittle LR. What is the translational efficacy of chemotherapeutic drug research in neuro-oncology? A systematic review and meta-analysis of the efficacy of BCNU and CCNU in animal models of glioma. J Neurooncol 2009;91:117-25. Epub 2008 Sep 24

52. Moffat BA, Chen M, Kariaapper MS, et al. Inhibition of vascular endothelial growth factor (VEGF)-A causes a paradoxical increase in tumor blood flow and up-regulation of VEGF-D. Clin Cancer Res 2006;12:1525-32

53. Mathieu V, De Nève N, Le Mercier M, et al. Combining bevacizumab with temozolomide increases the antitumor efficacy of temozolomide in a human glioblastoma orthotopic xenograft model. Neoplasia 2008;10:1383-92

54. McConville P, Hambardzumyan D, Moody JB. Magnetic resonance imaging determination of tumor grade and early response to temozolomide in a genetically engineered mouse model of glioma. Clin Cancer Res 2007;13:2897-904

55. Breton E, Goetz C, Kintz J, et al. In vivo preclinical low-field MRI monitoring of tumor growth following a suicide-gene therapy in an orthotopic mice model of human glioblastoma. C R Biol 2010;333:220-25. Epub 2010 Jan 25.

56. Huszthy PC, Immervoll H, Wang J. Cellular effects of oncolytic viral therapy on the glioblastoma microenvironment. Gene Ther 2010;17:202-16. Epub 2009 Oct 15

57. Heckl S, Pipkorn R, Nägele T, et al. Molecular imaging: bridging the gap between neuroradiology and neurohistology. Histol Histopathol 2004;19:651-68

58. de Backer ME, Nabuurs RJ, van Buchem MA, et al. MR-based molecular imaging of the brain: the next frontier. AJNR Am J Neuroradiol 2010;31:1577-83. Epub 2010 Sep 23

59. Jacobs AH, Dittmar, C, Winkeler A, et al. Molecular imaging of gliomas. $\mathrm{Mol}$ Imaging 2002;1:309-35

60. Chirasani SR, Markovic DS, Synowitz M, et al. Transferrin-receptor-mediated iron accumulation controls proliferation and glutamate release in glioma cells. J Mol Med (Berl) 2009;87:153-67. Epub 2008 Dec 9

61. Saxena V, Gonzalez-Gomez I, Laug WE. A non-invasive, in vivo technique for monitoring vascular status of glioblastoma during angiogenesis. Technol Cancer Res Treat 2007;6:641-50

62. Griffin JL, Kauppinen RA. Tumour metabolomics in animal models of human cancer. J Proteome Res 2007;6:498-505 